\title{
Industrieforum - Industrial Forum
}

Deutsche Krebshilfe 1990 mit bestem Ergebnis seit Gründung

Förderungsschwerpunkte: neue Bundesländer und Knochenmark-Fremdspenderregister

Die Deutsche Krebshilfe konnte im Jahr 1990 mit Einnahmen in Höhe von DM 51,7 Mio das beste Ergebnis seit ihrer Gründung verzeich-nen, ein Resultat, «das uns mit Dankbarkeit erfüllt», wie der Vor-standsvorsitzende der Deutschen Krebshilfe, Dr. Helmut Geiger, auf der Jahrespressekonferenz in Bonn sagte.

Insgesamt 81 neue Projekte mit einem Förderungsvolumen von DM 46 Mio hat die Deutsche Krebshilfe im letzten Jahr bewilligen können, womit sich die Gesamtzahl aller bisher geförderten medizinisch-wis-senschaftlichen und sozialen Projekte auf 643 erhöht. Die Arbeitsschwerpunkte der Organisation lagen im vergangenen Jahr vor allem bei Unterstützungsmaßnahmen für die neuen Bundesländer und dem Aufbau eines nationalen Knochenmark-Fremdspen-derregisters. In den neuen Bundesländern, so Geiger, werde die Deutsche Krebshilfe beim Aufbau von Strukturen helfen, die eine optimale Bekämp-fung der Krebskrankheiten und eine gute Versorgung Krebskranker sicherstellen sollen. Für derartige Strukturverbesserungen habe der Vorstand der Deutschen Krebshilfe bis zum Jahresende 1990 insgesamt DM 10 Mio bereitgestellt. Für die neuen Bundesländer seien bisher schwerpunktmäßig vier Programme vorgesehen: Die Einrich-tung eines Innerdeutschen Hospitationsprogrammes, den Aufbau von Tumorzentren, die Ausstattung von Kinderkliniken mit hochauflösen-den Computersonographiegeräten und die Unterstützung von Selbst-hílfegruppen nach Krebs. Um möglichst vielen Leukämiepatienten eine realistische Chance zum Weiterleben zu geben, setzt sich die Deutsche Krebshilfe verstärkt für den zügigen Ausbau einer nationalen Knochenmark-Fremdspender-datei ein. Im Sinne einer Anschubfinanzierung stellte sie im Jahre 1990 dafür rd. DM 5 Mio bereit.

Mit zusätzlich DM 5 Mio wird die Deutsche Krebshilfe die finanzielle Grundlage schaffen, bis Ende dieses Jahres über eine Knochenmark-Fremdspenderdatei zu verfügen, in der rund 50000 freiwillige poten-tielle Spender mit einer Teiltypisierung registriert sein werden. Die weiteren Förderungsschwerpunkte der Organisation lagen im vergangenen Jahr unter anderem bei innovativen Forschungsprojek-ten auf dem Gebiet der Zell- und Molekularbiologie, bei Maßnahmen in der Schmerz- und Palliativmedizin sowie in der Nachsorge. Im Bereich der pädiatrischen Onkologie förderte die Deutsche Krebshilfe u. a. eine Pilotstudie zur Früherkennung einer Nervenkrebser-krankung im Säuglingsalter. Diese Studie ist das erste Früherken-nungsprogramm für eine bösartige Krebserkrankung bei Kindern in Deutschland. Deutsche Krebshilfe e. V. Thomas-Mann-Straße 40 Postfach 1467 D-5300 Bonn 1 (BRD) Telefon: 0228/7299026

Pharma-Unternehmen spendet Deutscher Hospizhilfe DM 100.000

Strahlende Gesichter gab es, als Renate Wiedemann, Journalistin, Initiatorin und Präsídentin der Deutschen Hospizhilfe, in diesen Tagen von Sigrid Kartschewski, Marketing-Leiterin des Pharma-Unternehmens Mundipharma in Limburg, offiziell einen Scheck über DM 100.000 erhielt. Das Geld - immerhin die größte Spende, die der gemeinnützige 
Renate Wiedemann (links), Deutsche Hospizhilfe, Buchholz, und Sigrid Kartschewski, Mundipharma, Limburg

Verein, der seine gesamte Arbeit ohne jegliche staatliche Unterstützung finanzieren muß, in den drei Jahren seines Bestehens bisher erhalten hat - stammt aus einer Umfrage, die Mundipharma 1990 unter einigen tausend Medizinern zu Problemen bei der Behandlung chronischer

Schmerzen gestartet hatte. Pro zurückgesandtem Frage-bogen gab es eine Spende von DM 100 für die Arbeit der Deutschen Hospizhilfe, zu deren wichtigsten Anliegen es gehört, den HospizGedanken - möglichst liebevoll begleitetes Leben in vertrauter Umge-bung bis zuletzt - in Deutschland bekanntzumachen und über eine ausreichende Schmerzbehandlung Schwerstkranker - vor allem Krebs- und Aidspatienten - mit Morphin aufzuklären. Sigrid Kartschewski, die die Fragebogen-Aktion und die damit ver-bundene Förderung der Deutschen Hospizhilfe initiierte, erklärte bei der kleinen Feierstunde im Maritim-Hotel in Bad Salzuflen die thera-peutischen Möglichkeiten von oralem, retadiertem Morphin: «Bei nur zweimaliger Tabletteneinnahme nach festem Zeitschema wird eine gleichmäßige, langanhaltende Schmerzfreiheit erreícht.» Die Folge: Viele Kranke, die oft nur noch mit ihren Schmerzen beschäftigt sind und keine Lust am Leben mehr haben, erholen sich in der ersten Tagen der Medikamenteneinnahme, gewinnen bei absoluter Bewußt-seinsklarheit neuen Lebensmut, und leben oft auch noch deutlich länger, als von ihren Ärzten vorher prognostiziert. Und das alles ohne Suchtentwicklung.

«Und um möglichst vielen Schwerkranken mit Hilfe moderner Schmerztherapie ein erträgliches Leben bis zum letzten Tag zu erlau-ben und hierüber entsprechende Aufklärung zu leisten, darum haben wir die Arbeit der Deutschen Hospizhilfe mit dieser Summe unter-stützt», erklärte Sigrid Kartschewski. «Denn keine Institution sonst in Deutschland setzt sich öffentlich so für die Belange Sterbender und ihrer Angehörigen ein, wie die Deutsche Hospizhilfe. Und wir können nur hoffen, daß auch andere - Firmen, Institutionen, Burger - unse-rem Beispiel folgen werden.» Die Deutsche Hospizhilfe verschickt gegen Vorauszahlung des Selbst-kostenpreises von DM 10 eine Informationsmappe mit Unterlagen zum Hospiz-Gedanken, Adressen von Hospiz-Initiativen und Bro-schüren wie «Zu Hause sterben» und «Schmerztherapie bei sterben-den Menschen - die orale Morphintherapie in der Hand des Haus-arztes» von ihrem Gründungsmitglíed Professor Dr. med. Johann-Christoph Student aus Hannover, deren Entwicklung vom Bundes-

bildungsministerium und dem Niedersächsischen Ministerium für Wissenschaft und Kunst gefördert wurde.

Deutsche Hospizhilfe e.V.

Reit 25

D-2110 Buchholz (BRD)

370

Industrieforum $\mathbf{a}$ Industrial Forum

Das erste natürliche Interferon Alpha-Präparat

Die Firma Dr. Rentschler Arzneimittel GmbH \& Co., Laupheim, hat von der Firma BioNative, Umea, Schweden, die Vertriebsrechte für Deutschland und einige europäische Lander von natürlichem Interferon alpha erworben.

Das hochgereinigte natürliche Interferon alpha (Alphaferon $\left.{ }^{\circledR}\right)$ wird von Rentschler in Deutschland klinisch geprüft. Im Gegensatz zu den Glykoproteinen Interferon beta und gamma, bei denen es sich um Monosubstanzen handelt, ist natürliches Interferon alpha ein Gemisch aus ca. 20 Einzelproteinen bzw. -glykoproteinen (Subtypen). Die weltweit im Handel befindlichen 
gentechnischen Alpha-Interferone enthalten demgegenüber nur einen einzigen Subtyp von Interferon alpha. Es gibt eine Reihe von Hinweisen, daß das natürliche Interferon alphaSubtypengemisch nicht nur seltener zur Resistenzentwick-lung führt, sondern auch wirksamer ist als die rekombinanten Interfe-ronmonosubstanzen.

Dies gilt z. B. für die Therapie des Nierenzellkarzinoms und des Karzinoids. Alphaferon ${ }^{\circledR}$ soil auch bei Patienten eingesetzt werden, die gegen andere Interferone neutralisierende Antikörper gebildet haben.

In Schweden ist das natürliche Interferon alpha zur Behandlung des metastasierenden NierenzellKarzinoms zur Zulassung eingereicht. Mit der vereinbarten Kooperation erweitert Rentschler neben Fibla-feron ${ }^{\circledR}$ sowie Fiblaferon ${ }^{\circledR}$ Gel (natürliches Interferon beta) und Poly-feron ${ }^{\circledR}$ (Interferon gamma rekombiniert) sein Angebot und sein medi-zinisches Know-how auf dem Interferon-Sektor. Rentschler ist damit weltweit das einzige pharmazeutische Unterneh-men, das alle Interferonarten im Programm hat.

Institut für Medizinische Kommunikation Rosenkavalierplatz 8 D-8000 München 81 (BRD) Myeloregeneration gegen Neutropenie und Infektion

Eine der häufigsten Nebenwirkungen einer aggressiven zytostatischen Tumortherapie ist die Neutropenie. Dies kann einerseits ein erhöhtes Sepsisrisiko für den Patienten und andererseits die Notwendigkeit einer Dosisreduktion der eingesetzten Zytostatika bedeuten. Dadurch kann der Erfolg der Chemotherapie entscheidend beeinträchtigt werden. Dieser Problemkreis läßt sich mit dem bald auch in Deutschland zur Verfügung stehenden hämatopoetischen Wachstumsfaktor G-CSF (Neupogen $\AA /$ Amgen-Roche) durchbrechen.

Im Rahmen eines Symposiums am 8. Juni 1991 in Bonn unter'dem Vorsitz von Prof. R. Mertelsmann, Freiburg, und Prof. D. Hoelzer, Frankfurt/Main, wurden Wirkprinzip sowie klinische Erfahrungen aus den USA und verschiedenen europäischen Ländern vorgestellt und diskutiert.

G-CSF stimuliert als spezifisch wirkender Wachstumsfaktor selektiv die schon reiferen Vorläuferzellen für neutrophile Granulozyten. Nach Gabe von Neupogen kommt es innerhalb von vier bis fünf Stunden zu einem schnellen und beträchtlichen Anstieg der Granulo-zytenzahl. Der Effekt von Neupogen ${ }^{\circledR}$ ist dosisabhängig. Die empfoh-lene Dosierung beträgt $5 \mu \mathrm{g} / \mathrm{kg}$ Körpergewicht/Tag, als subkutane Bolusgabe, für zehn bis 14 Tage nach der Zytostatikatherapíe. Die aufgrund der Stimulation gebildeten reifen, neutrophilen Granulozyten besitzen eine normale bzw. sogar verstärkte Fähigkeít zur Phago-zytose und Chemotaxis.

In plazebokontrollierten klinischen Studien in den Vereinigten Staaten sowie in verschiedenen europäischen Ländern konnten für Neupogen ${ }^{\circledR}$ folgende Vorteile dokumentiert werden:

- Eine signifikante Verringerung der Inzidenz, des Schweregrades sowie der Dauer der durch Zytostatika induzierten, febrilen Neutropenie;

eine Verminderung der Notwendigkeit stationärer Behandlungen; ein geringerer Bedarf an parenteral zu verabreichenden Antibioti-ka. Geprüft wurde Neupogen ${ }^{\circledR}$ bislang u.a. bei Patienten mit akuter lymphoblastischer Leukämie (ALL), bei hochmalignen Non-Hodg-kin-Lymphomen, gynäkologischen Tumoren sowie bei Patienten mit kleinzelligem bzw. nicht-kleinzelligem Bronchialkarzinom. Wie CA. Meanwell, Basel, konstatierte, ist Neupogen ${ }^{\circledR}$ gut verträg-lich. Als häufigste Nebenwirkung werden Knochenschmerzen angege-ben. Ein das Tumorwachstum stimulierender Effekt wurde in vivo nicht beobachtet. 
Der Neupogen ${ }^{\circledR}$-Effekt bleibt bei wiederholter Applikation auch über mehrere ChemotherapieZyklen erhalten, wobei die verstärkte Bil-dung von neutrophilen Granulozyten nicht auf Kosten der Erythrozy-ten-, Thrombozyten- und/oder Lymphozytendifferenzierung im Kno-chenmark erfolgt. Die Integration von Neupogen ${ }^{\circledR}$ in das jeweilige Chemotherapie-Protokoll der onkologischen Patienten ist in der Re-gel problemlos möglich.

Die derzeit vorliegenden Ergebnisse deuten darauf hin, daß unter Neupogen ${ }^{\circledR}$ die Chemotherapie in der überwiegenden Zahl der Fälle nach dem vorgegebenen Therapieschema durchgeführt werden kann. Momentan wird in klinischen Studien weiter geprüft, ob durch Neupogen ${ }^{\circledR}$ auch die Zytostatika-Dosen in den jeweiligen Zyklen erhöht, bzw. das behandlungsfreie Intervall zwischen den einzelnen Thera-piephasen im Sinne einer potentiell kurativen Intention verkürzt werden könnte.

Institut für medizinische Kommunikation Dr. med. Michael Fischer Rosenkavalierplatz 8 D8000 München 81 (BRD)

Curt Engelhorn vollendet sein 65. Lebensjahr

Am Samstag, 25. Mai 1991, feierte Curt Engelhorn, Aufsichtsratsvor-sitzender der Boehringer Mannheim GmbH und Chairman des Board of Directors der Corange Ltd., den 65. Geburtstag. Sein Name ist untrennbar verbunden mit dem beispiellosen Aufschwung von Boehringer Mannheim, das er seit den 50er Jahren vom mittelständigen Unternehmen mit einem Umsatz von 50 Millionen Mark zu einer internationalen Firmengruppe mit globaler Präsenz und einem Umsatz von 4 Milliarden Mark geformt hat. Weltweit sind unter dem Dach der Boehringer Mannheim-Gruppe etwa 20.000 Mitarbeiterin-nen und Mitarbeiter tätig.

Curt Engelhorn brachte für seine führende Position bei Boehringer Mannheim geeignete Voraussetzungen mit. Hier die wichtigsten Sta-tionen seines Lebens:

Am 25. Mai 1926 in München geboren, siedelte er nach dem Abitur 1947 nach Austin (Texas) um, beendete 1951 das Chemical Engineer-ing-Studium an der University of Texas als Bachelor of Science, arbeitete zwei Jahre in der amerikanischen Pharmaindustrie, kehrte 1953 nach Deutschland zurück und wurde kurz darauf Direktionsassi-stent bei Boehringer Mannheim. Als solcher koordinierte er den Aufbau eines modernen Firmenimages mit u.a. neuem Warenzeichen, einheitlicher Packungsgestaltung und Neukonzipierung der gesamten Werbung.

Mit 34 Jahren wurde Curt Engelhorn 1960 Geschäftsführender Gesell-schafter, nachdem er 1956 stellvertretender und 1958 ordentlicher Geschäftsführer geworden war.

1964 wählten ihn die Mitglieder des Bundesverbandes der Pharmazeu-tischen Industrie (BPI) zum Vorsitzenden. Er übte dieses Amt nach zweimaliger Wiederwahl bis 1970 aus und blieb bis 1985 im Vorstand tätig. Parallel dazu nahm Engelhorn wichtige Funktionen im Verband der Chemischen Industrie (VCI) sowie im Institut der Deutschen Industrieforum $\square$ Industrial Forum 375

Industrie wahr und erhielt 1964 die Würde eines Ehrensenators der Universität Heidelberg, mit der Boehringer Mannheim bis in die Gegenwart rege Forschungsförderung und -austausch verbindet.

Boehringer Mannheim GmbH Kommunikation/Medien Sandhofer Straße 116 D-6800 Mannheim 31 (BRD) Tel: 0621/7592818 und 7593502 Telefax: 0621/7592902

Firmenzusammenführung Pfrimmer-Viggo GmbH \& Co. KG und Spectramed GmbH Die Intensivierung der Forschungsaktivitäten im Dienste des Men-schen und seiner Umwelt sowie die konsequente Durchsetzung einer weltweiten Strategie der BOC Health Care sind 
Anlaß für die Fusion der Unternehmen Pfrimmer-Viggo GmbH \& Co. KG, Erlangen und Spectramed GmbH, Düsseldorf, im weltweit zweitgrößten Markt für Medical-Produkte. Der Zusammenschluß der beiden Unternehmen unter dem Namen Pfrimmer-Viggo GmbH \& Co. KG mit Standort in Erlangen verfolgt neben umweltpolitischen und wirtschaftlichen Gesichtspunkten vor allem kundenorientierte Aspekte:

Herstellung einer breiten, anwenderorientierten und marktgerech-ten Produktpalette mit maximalem Qualitätsanspruch;

Verstärkung und Optimierung der Serviceleistungen für die Anwen-der unserer Produkte; Intensivierung unserer Anwenderberatung durch kompetente Mit-arbeiter mit SpezialistenKnow-how;

Initiierung einer ökologieorientierten, partnerschaftlichen Zusam-menarbeit zwischen Anwendern und unserem Unternehmen.

Das gemeinsame Leistungsprogramm umfaßt drei Bereiche: Bereich Medical-Produkte:

Periphervenöse, zentralvenöse und arterielle Zugangssysteme

Subkutane und implantierbare Kathetersysteme

Infusions-/Transfusionssysteme Bereich Medizintechnik:

Invasive Blutdrucküberwachungs-Systeme

Rechtsherz-Katheter-Systeme

Computer zur Erstellung des hämodynamischen Profills Bereich Wundversorgung:

Chirurgisches Nahtmaterial

Transplantate

Spezialprodukte für die Chirurgie

Pfrimmer-Viggo GmbH \& Co. KG

Langemarckplatz 3

Postfach 2880

D-8520 Erlangen (BRD)

Telefon: 09131/8101-0

Queen's Award für Zoladex

Die höchste britische Innovationsauszeichnung, der «Queen's Award for Technological

Achievement», ist jetzt für 1991 an ICI Pharmaceuticals für die Entwicklung des

Krebsmedikaments Zoladex verliehen worden. Wie die deutsche ICI Pharma am 25. April in Plankstadt mitteilte, fiel die Wahl auf Zoladex, weil das Präparat sowohl als Wirkstoff einen großen therapeutischen Fortschritt darstellt als auch von der Darreichungsform her: Zoladex wird einmal monatlich als Kleindepot injiziert, was die Compliance sichert. Das seit 1988 auch in Deutschland verfügbare Medikament ist ein

führendes Präparat bei Prostatakarzinom, wo seine Anwendung in vielen Fallen an die Stelle der bisher üblichen Operation getreten ist. In der Zwischenzeit ist es auch für Brustkrebs zugelassen und befindet sich in der klinischen Erprobung bei Endometriose und Uterusmyom. Nach Angaben von ICI Pharma werden 1991 rund 600 Millionen Mark für die Arzneimittelforschung ausgegeben werden, wovon ein großer Teil auf die Krebsforschung entfällt. Große Erwartungen in die Kom-plettierung der therapeutischen Palette gegen das Prostatakarzinom setzt ICI in das neue Antiandrogen Casodex, welches sich gegenwärtig in Phase II der klinischen Erprobung befindet. Für Nolvadex, das inzwischen weltweit meistangewendete Medikament gegen Brustkrebs, war ICI 1978 mit dem Queen's Award ausgezeichnet worden. Insgesamt konnte die Pharma-Sparte von ICI seit 1965 vier dieser Innovationspreise erringen.

Rückfragen an: 
ICI Pharma

Herrn Wenk

Otto-Hahn-Straße

D-6831 Plankstadt (BRD)

Tel.: 06202/82218

oder

Agentur für Dialog-Kommunikation GmbH (GPRA)

Herrn Cramer

Kommunikationsfabrik

Schmidtstraße 12

D-6000 Frankfurt/Main 1 (BRD)

Tel.: 069/75804-444, Telefax: 069/7384479

Bronchialelixier ERES durch ERES N ersetzt

Müller Göppingen hat sein Bronchialelixier ERES durch das Nachfol-gepräparat ERES N

ersetzt. ERES N ist ein pflanzliches Monopräpa-rat und enthält einen Fluidextrakt aus

Wollblumen (Verbasci flos) auf der Basis der Monographie der Komíssíon E.

Anwendungsgebiete von ERES N sind Katarrhe der Luftwege bei Kindern und Jugendlichen.

Wegen des hohen Wirkstoffgehaltes konnte der Packungsinhalt von bisher 250 auf $100 \mathrm{ml}$

verringert werden. Diese Packungsgröße ist für Behandlungszeiten von mehr als zwei Wochen ausreichend.

Chemisch-Pharmazeutische Fabrik Göppingen Carl Müller, Apotheker, GmbH u. Co. KG

Zeiss Mikroskop Standard 25:

Das Routinemikroskop, das mehr kann

Den «großen Bruder» der Mikroskop-Reihe Standard 20 stellt Carl Zeiss, Oberkochen, mit dem neuen Modell Standard 25 vor. Zeiss bietet damit ein Labormikroskop mit deutlich erweiterten Möglichkei-ten an. Durch auswechselbare Komponenten läßt sich die Mikroskop-reihe Standard 25 für viele Aufgaben im Bereich der biologischen und medizinischen Routine

anwendungsspezifisch ausrüsten. Mehr Flexibilität gegenüber dem Standard 20 bietet das

Mikroskop Standard 25 durch seinen größeren Objektivrevolver, der fünf Objek-tive aufnimmt.

Damit ist Platz für fein abgestufte Vergrößerungsrei-hen oder Spezialoptiken, die nur für bestimmte Aufgaben benötigt werden.

Mit der Reihe «A-Plan» steht ein neuer Objektivtyp zur Verfügung, der als Nachfolger der FAchromat-Objektive deutlich verbesserte Leistung aufweist. Die Bildfeldebnung der Objektive A-Plan erlaubt beispielsweise die exakte Differenzierung eines Blutausstrichs über das gesamte Sehfeld.

376

Industrieforum $\mathbf{a}$ Industrial Forum

Wechselbar ist beim Mikroskop Standard 25 auch der Kondensor.

Hellfeld- und Dunkelfeldkondensoren sowie ein Revolverkondensor

für Hellfeld und Phasenkontrast bieten das jeweils passende Gegen-

stück zum gewählten Objektiv.

Für die Aufnahme von Präparaten verschiedener Art und Größe ist der Kreuztisch mit seiner großen Auflage und dem weiten Verstellbereich vorbereitet.

Das neue Zeiss Routinemikroskop bietet auch die Möglichkeit zur 
Untersuchung von Biokristallen (z.B. zur Gicht-Pseudogicht-Differenzierung). Hierfür läßt sich eine spezielle Analysator/Lambdaplatten-Kombination in das Stativ integrieren.

Carl Zeiss

Postfach 1369/1380

D-7082 Oberkochen

Tel.: 07364/20-0, Telefax: 07364/6808

ILCO-Stoma-Information in Jena

Am Samstag, den 19. Oktober 1991, findet in Jena eine ГLCO-Veranstaltung mit aktuellen Informationen zu den Stomaoperationen und zur Stomaversorgung statt. Es ist dies die 3. ILCOStoma-Information in den neuen Bundesländern. Erwartet werden über 200 Teilnehmer aus den Bereichen Medizin, Krankenpflege, Sozialarbeit, Stomaversorgungs-Handwerk und -Handel sowie insbesondere Stomaträger mit Angehörigen.

Deutsche ILCO Postfach 1265 D-8050 Freising (BRD) Tel. 08161/84909/11

Neue Aspekte in der Therapie yon Hodentumoren

Medizinische Hochschule Hannover, 6. und 7. Dezember 1991 Leitung: Prof. Dr. L. Weißbach, Prof. Dr. H.-J. Schmoll

Ein Symposium der interdisziplinären »Arbeitsgruppe Hodentumoren «

der Arbeitsgemeinschaft Internistische Onkologie (AIO) Leitung: Prof. D. K. Hossfeld der Arbeitsgemeinschaft Urologische Onkologie (AUO) Leitung: Prof. Dr. H. Huland der Arbeitsgemeinschaft Radiologische Onkologie (ARO) Leitung: Prof. Dr. R. Sauer Um die Einsendung von Abstracts zur Präsentation als Vortrag oder Poster wird herzlich gebeten. Deadline hierzu ist der 15.9.1991. Das Programm-Komitee wählt die eingereichten Abstracts zum Vortrag oder für die Postersession aus.

Information:

Frau S. Dröge

Abt. Hämatologie/Onkologie, Medizinische Hochschule Hannover

Konstanty-Gutschow-Straße 8, D-3000 Hannover 61

Tel. 05 11/5 3240 77, Fax 05 11/5323691 